\title{
AUTOMATIC RECOGNITION OF ADHESION STATES USING AN EXTREME LEARNING MACHINE
}

\author{
Changfan Zhang, ${ }^{*}$ Xiang Cheng, ${ }^{*}$ Jing He, ${ }^{*}$ and Guangwei Liu*
}

\begin{abstract}
In this study, the use of an extreme learning machine (ELM) for automatic identification of the adhesion state is investigated. The influence of different activation functions and the number of neurons in the hidden layers on the recognition performance is investigated. This study aims to select a better activation function and construct an approach for the adhesion state recognition by using an ELM. Monitoring data on the adhesion characteristics of a heavy-duty locomotive are used to fabricate the recognition model. Comparing with the backpropagation (BP) neural network and a BP optimized algorithm, the experimental results show that our ELM recognition method has a faster training speed and higher recognition accuracy than the other two approaches.
\end{abstract}

\section{Key Words}

Adhesion control, extreme learning machine, automatic recognition, machine learning, neural network

\section{Introduction}

Precise automatic recognition of the wheel-rail adhesion state is an important prerequisite for adhesion control [1]. Many recognition methods have been used in traditional approaches to automated adhesion control. The adhesion state has been estimated using wheel encoders, inertial measurement, and global positioning system (GPS) [2]. Operating data from a measuring device mounted on a locomotive wheel and filtering processing have been used to estimate the current state of adhesion [3]. An alternative strategy estimated the adhesion coefficient from the creep velocity and used this to determine the current state of adhesion [4]. There are numerous drawbacks to these methods. All identify the adhesion state only after a fault has occurred; precise analysis of the mechanisms involved is difficult; and hardware installation is both expensive and challenging to realize in practical engineering applications.

* College of Electrical and Information Engineering, Hunan University of Technology, People's Republic of China; e-mail: zhangchangfan@263.com, chengxiang@stu.hut.edu.cn, hejing@263.com,mrliugw@163.com

Corresponding Author: Jing He

Recommended by Prof. Chaomin Luo

(DOI: 10.2316/Journal.206.2017.2.206-5067)
The change in adhesion state is a complex process, which is affected by multiple factors, producing a complex nonlinear relation between factors and outcomes. Fault prediction and analysis is particularly challenging. Machine learning algorithms can investigate the intrinsic relations among factors using input information and identify mappings in seemingly unrelated data [5]. This is particularly advantageous when addressing such complex problems.

As machine learning has developed, a range of intelligent methods have been applied to adhesion control. Optimized recurrent neural networks have been used to optimize the parameters of the adhesion controller in locomotive adhesion [6]. In [7], a backpropagation (BP) neural network was used to estimate the reference speed of a vehicle antilock brake system (ABS) system, improving the accuracy of parameter acquisition. A method for predicting wheel slip based on a support vector machine and an adhesion theory was presented in [8]. Adhesion state recognition based on traditional neural network and support vector machine has avoided detailed analysis of the mechanism of slippage that traditional methods find challenging, but outstanding problems with this approach include a slow learning speed [9], [10] and difficulty in adjusting the parameters.

The extreme learning machine (ELM) is a new type of learning algorithm based on a single-hidden layer feedforward neural network [11]. In the training process, this algorithm randomly generates the connection weights between the input and hidden layers and the threshold of the hidden layer neuron; also, it does not need to be adjusted repeatedly. By setting the number of neurons in the hidden layer, a unique optimal solution can be obtained. The ELM offers a faster learning speed and better generalization, thereby avoiding the defects of traditional learning methods, and has attracted increasing research attention [12]-[15]. However, it has not yet been applied to adhesion state recognition.

The rest of this paper is organized as follows. Section 2 introduces the adhesion characteristics. Section 3 discusses our construction of the adhesion state recognition model in ELM. Section 4 introduces our experimental comparison of three recognition methods and presents the results. Section 5 gives our conclusions. 


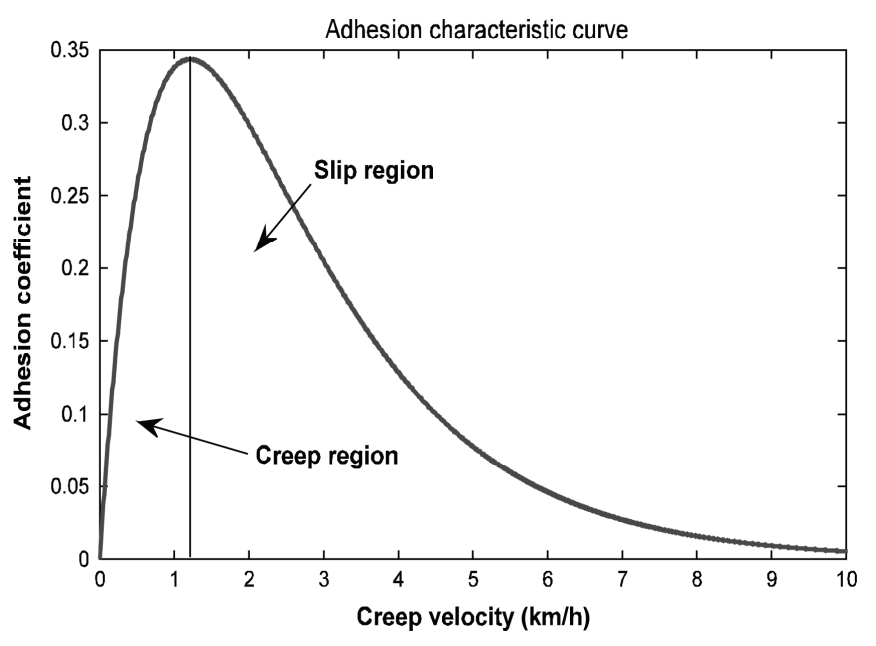

Figure 1. Adhesion characteristic curve.

\section{Adhesion Characteristics}

Adhesion is essentially an elastic contact interaction between a wheel and rail. When subjected to tangential traction, the wheel pair rolls forward and the resulting pressure causes deformation to occur between the wheel and rail. Simultaneously, the positive pressure that the car body imposes on the rail keeps the contact surface between the wheel and rail relatively stable. This phenomenon is called "adhesion". In this process, the wheel pair speed $v_{d}$ is greater than the speed of the train $v_{t}$ and the difference between the two is defined as the creep speed $v_{s}$. The ratio of the tangential traction force $F_{u}$ acting on the wheel and the axle load $W \cdot g$ is defined as the adhesion coefficient $\mu$. The related expressions are as follows [16]:

$$
\begin{aligned}
v_{s} & =v_{d}-v_{t} \\
v_{d} & =\omega \cdot R \\
\mu & =\frac{F_{u}}{W \cdot g}
\end{aligned}
$$

where $R$ is the radius of the locomotive wheel pair, $\omega$ is the angular velocity of the wheel pair, $v_{t}$ is the speed of the train, $W$ is the axle load, $g$ is the acceleration owing to gravity, and $F_{u}$ is the tangential traction. From (3), it can be seen that, with a given axle load, the locomotive traction force is proportional to the adhesion coefficient, so the adhesion coefficient can represent the change in traction force. Numerous experimental studies and theoretical analyses have shown that the adhesion characteristics can be characterized by the relation between the adhesion coefficient $\mu$ and the creep speed $v_{s}$. The adhesion characteristic curve is shown in Fig. 1 [17].

It can be seen from Fig. 1 that the adhesion characteristic curve reaches a peak, the curve splits into two regions: a creep region and a slip region. If the locomotive enters the slip region, there is a danger of slipping or even of derailment. So timely and accurate identification of the adhesion state is important when predicting failure. However, there is insufficient time to prevent slipping when the operating point of the locomotive is close to the peak point, even if it is accurately predicted.

In this study, we proposed an ELM-based recognition method to have an early prediction of a fault from refinement of the adhesion characteristics. The accuracy and real-time performance of our approach are investigated experimentally and compared with other methods.

\section{Recognition Model of the Adhesion State by Using an ELM}

\subsection{Extreme Learning Machine}

An ELM has a three-layer network structure: an input layer, a hidden layer, and an output layer, with a connecting structure between the layers [18]. Suppose that the input layer has $n$ neurons, the hidden layer has $l$ neurons, the output layer has $m$ neurons, and the connection weight matrix between the input and hidden layer is $w$. The activation function of the hidden layer neuron is $g(x)$, and the output of the ELM $T$ can be defined as [19]:

$$
\begin{gathered}
T=\left[t_{1}, t_{2}, \ldots, t_{Q}\right]_{m \times Q} \\
t_{j}=\left[\begin{array}{l}
t_{1 j} \\
t_{2 j} \\
\vdots \\
t_{m j}
\end{array}\right]_{m \times 1}=\left[\begin{array}{l}
\sum_{i=1}^{l} \beta_{i 1} g\left(w_{i} x_{j}+b_{i}\right) \\
\sum_{i=1}^{l} \beta_{i 2} g\left(w_{i} x_{j}+b_{i}\right) \\
\vdots \\
\sum_{i=1}^{l} \beta_{i m} g\left(w_{i} x_{j}+b_{i}\right)
\end{array}\right]_{m \times 1}(j=1,2, \ldots, Q)
\end{gathered}
$$

where $w_{i}=\left[\omega_{i 1}, \omega_{i 2}, \ldots, \omega_{i n}\right] ; x_{j}=\left[x_{1 j}, x_{2 j}, \ldots, x_{n j}\right]^{T}, \omega_{j i}$ represents the connection weight between the $i$ th neuron of the input layer and the $j$ th neuron of the hidden layer, $Q$ is the number of samples, $\beta_{j k}$ represents the connection weight between the $j$ th neurons of the hidden layer and the $k$ th neuron of the output layer, $b$ is the threshold of the hidden layer neuron, $T^{\prime}$ is the transposition of matrix $T, H$ is the hidden layer of the output matrix of the neural network, and $\beta$ is the weighted matrix by which the hidden layer connects with the output layer. Therefore, the output of ELM $T^{\prime}$ from (5) can be simplified as follows:

$$
H \beta=T^{\prime}
$$

The difference between the ELM and traditional neural network is its premise of an infinite differentiable activation function. The parameters of an ELM do not need to be adjusted in their entirety. Instead, $w$ and $b$ are randomly selected and unchanged before and during the training process. Only adjusting the number of neurons in the hidden layer can obtain a unique solution from (6): $\hat{\beta}=H^{\dagger} T^{\prime}$, where $H^{\dagger}$ is the generalized inverse of $H$ [20]. Therefore, ELM has fewer parameters, a faster learning speed, and a more generalized performance than the traditional neural network. 


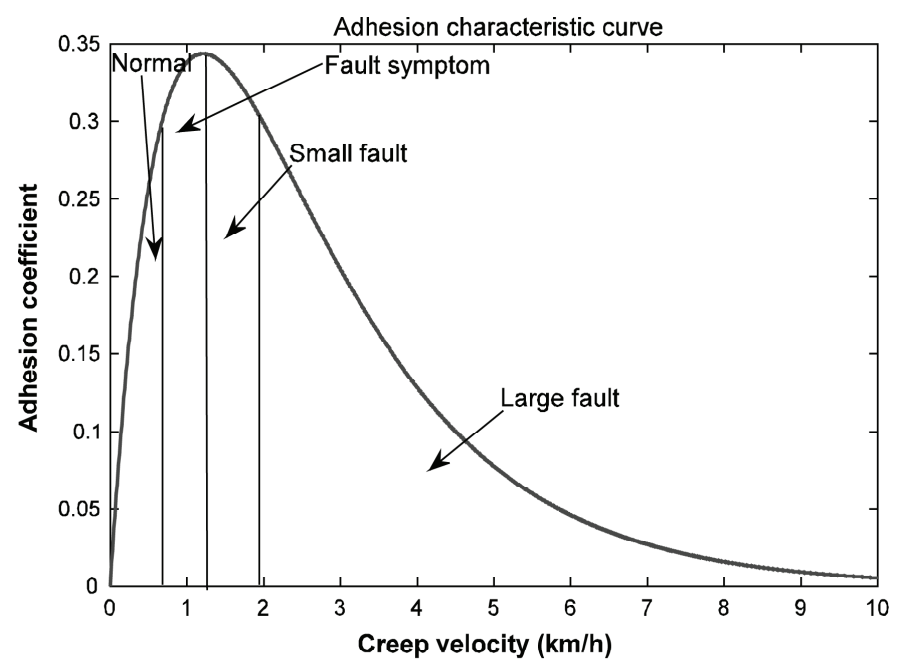

Figure 2. The recognition region.

\subsection{Recognition Mode}

To allow timely prediction of locomotive slipping, more accurate recognition of the adhesion state between the wheel and rail is needed. For practical and accurate estimation of the adhesion state, the adhesion coefficient $\mu$ and creep velocity $v_{s}$ are used as the inputs to the recognition model, and the adhesion characteristic curve is subdivided into four regions: normal $(\mathrm{N})$, fault symptom (N1), small fault (F1), and large fault (F2). In the ELM model, $\{i=1,2$, $3,4\}$ represented the four regions and the number of neurons in the output layer of the ELM is set to one. The division of the recognition region is shown in Fig. 2.

\subsection{Activation Function}

In this study, four types of activation function are compared: a sigmoid function, a sin function, the hard-limit transfer function, and the radial basis function (RBF):

$$
\begin{aligned}
& \text { Sigmoid : } g\left(w_{i} x+b_{i}\right)=\frac{1}{1+\left(\exp \left(-w_{i} x+b_{i}\right)\right)} \\
& \text { Sin : } g\left(w_{i} x+b_{i}\right)=\sin \left(w_{i} x+b_{i}\right) \\
& \text { Hard-limit : } g\left(w_{i}+b_{i}\right)= \begin{cases}1, & w_{i} x+b_{i} \geq 0 \\
2, & w_{i} x+b_{i}<0\end{cases} \\
& \mathrm{RBF}: g\left(w_{i}, b_{i}, x\right)=\exp \left(-\frac{\left\|x-w_{i}\right\|^{2}}{b_{i}^{2}}\right)
\end{aligned}
$$

\subsection{Recognition Model}

The recognition process of the adhesion state based on the ELM is shown in Fig. 3 and is conducted as follows:

1. Randomly select training and test sets from the sample data.

2. Determine the number of hidden layer neurons.

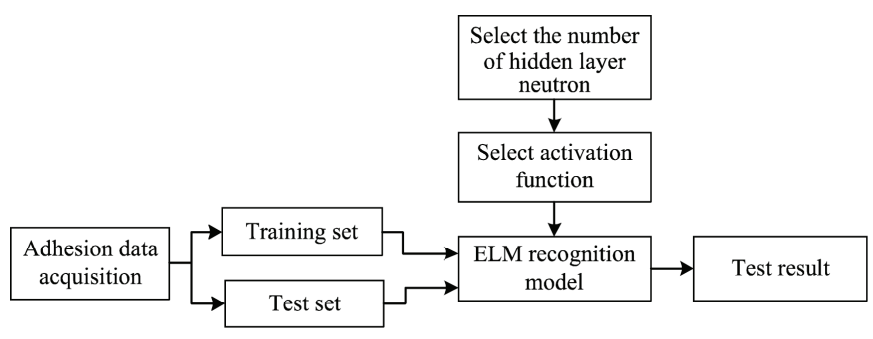

Figure 3. The recognition process.

Table 1

State Type

\begin{tabular}{|l|c|ccc|}
\hline \multicolumn{2}{|l|}{ State Type } & State Encoding \\
\hline Normal & (N0) & 0 & 0 & 0 \\
\hline Failure symptom & (N1) & 0 & 0 & 1 \\
\hline Tiny fault & (F1) & 0 & 1 & 0 \\
\hline Large fault & (F2) & 1 & 0 & 0 \\
\hline
\end{tabular}

3. Select the activation function of the ELM.

4. Train the ELM model and conduct state recognition of the test samples.

\section{Simulation Study}

In all, 323 data sets are collected from an engineering site, 279 of them are used as the training set and the rest are as the test set. To meet the engineering requirements, white Gaussian noise is added to the test data, with the mean being 0 and the variance being 0.02 .

For performance comparison with the ELM, a BP neural network and an optimized BP neural network based on a genetic algorithm (GA-BP) are also used as state recognition models. The experiments are conducted on MATLAB R2015b, with a dual core $1.8 \mathrm{GHz} \mathrm{CPU}, 4 \mathrm{~GB}$ of memory PC.

\subsection{Simulation of the BP Neural Network}

Neural network is a classic intelligent algorithm that is widely used in optimization and identification research [21]-[23]. This section presents the use of the BP neural network for conducting an experimental research. As shown in Table 1, the empirical formula $l=2 n+1$ is used to simulate the BP neural network, where $l$ is the number of hidden layer nodes and $n$ is the dimension of the feature vector. The hidden layer node number of the $\mathrm{BP}$ neural network is set to 5 , the adhesion coefficient $\mu$ and creep velocity $v_{s}$ are the input variables, and the state type used the binary encoding form. This represented a BP neural network with a $2 \times 5 \times 3$ structure, with accuracy set to 0.01 and the default values used for the other parameters. A schematic diagram of the structure is shown in Fig. 4, the simulation error curves are shown in Fig. 5, and the recognition results are shown in Table 2. 


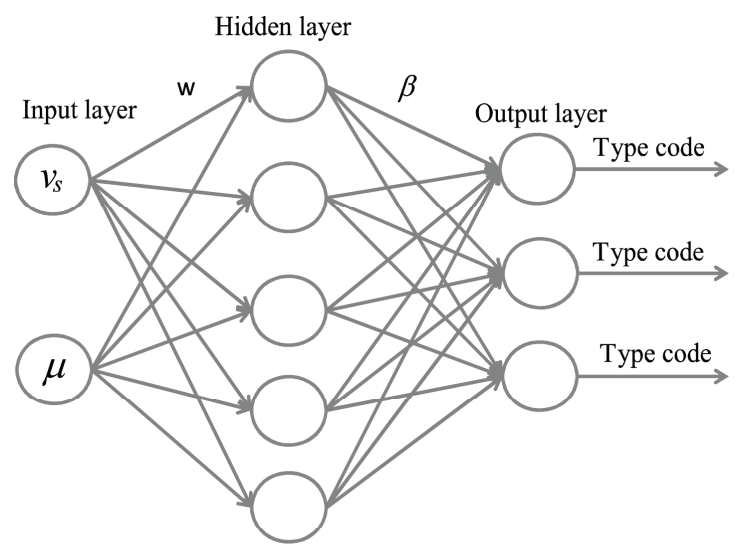

Figure 4. Schematic diagram of the structure of BP neural network.

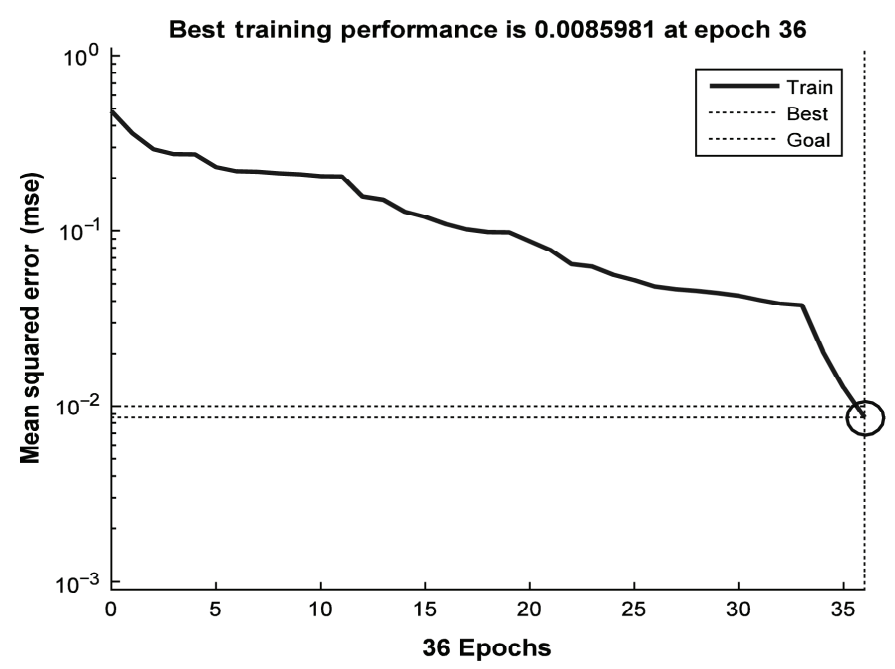

Figure 5. Simulation error curves.

Table 2

Recognition Results

\begin{tabular}{|c|c|c|c|c|c|c|}
\hline \multirow{2}{*}{$\begin{array}{l}\text { Fault } \\
\text { Types }\end{array}$} & \multirow{2}{*}{$\begin{array}{c}\text { Number of } \\
\text { Samples }\end{array}$} & \multicolumn{4}{|c|}{$\begin{array}{l}\text { The Result of } \\
\text { Recognition }\end{array}$} & \multirow{2}{*}{$\begin{array}{l}\text { Accuracy } \\
\text { Rate (\%) }\end{array}$} \\
\hline & & N0 & N1 & F1 & F2 & \\
\hline N0 & 6 & 5 & 1 & 0 & 0 & 83.33 \\
\hline N1 & 7 & 0 & 4 & 3 & 0 & 57.14 \\
\hline F1 & 9 & 1 & 0 & 7 & 1 & 77.78 \\
\hline $\mathrm{F} 2$ & 22 & 0 & 0 & 1 & 21 & 95.45 \\
\hline
\end{tabular}

Because the BP neural network used in this experiment has a single-hidden layer structure, the weights and thresholds are not optimized, the randomness of the initial values of the network parameters is greater, and the recognition results are poor. Increasing the depth of the network and optimizing its weight, threshold, and network structure would yield better recognition results.

\subsection{Simulation of the BP Neural Network Based on a Genetic Algorithm}

A GA is a heuristic stochastic optimization algorithm that is based on an evolutionary approach [24]. GA is widely used for algorithm optimization [25], [26]. Through the adaptive operations of selection, crossover, and mutation, an optimal solution can be converged upon in any domain that contains such an optimal solution [27]. The traditional BP neural network has drawbacks of slow convergence, oscillation in convergence, and convergence on local optima. A GA can be used to compensate for these defects. The optimization of a BP neural network in this way involves population initialization, calculation of a fitness function, selection, crossover, and mutation. We discuss these in turn.

\subsubsection{Population Initialization}

In this study, a GA chromosome code with a binary form is used. The recognition model has two inputs, three output layer nodes, and five hidden layer nodes. The weight from the input layer to the hidden layer is $2 \times 5 w_{i j}$, the weight from the hidden layer to the output layer is $5 \times 3 w_{j k}$, the hidden layer had five thresholds, and the output layer had three. The number of optimization parameters is therefore $2 \times 5+5 \times 3+5+3=33$. Encoding of both setting weight and threshold is done with 10 bit binary numbers, giving a binary code length of 330 .

\subsubsection{Fitness Function}

To keep the error between the predictive value and the expected value of the BP neural network within a reasonable range, the sum of the absolute values of the training data prediction error is used as the individual fitness value $F$. This is given as follows: $F=k\left(\sum_{i=1}^{n} \operatorname{abs}\left(Y_{i}-T_{i}\right)\right.$, where $n$ is the number of output nodes, $Y_{i}$ is the expected output of the $i$ th node of the BP neural network, $T_{i}$ is the predictive output of the $i$ th node, and $k$ is the coefficient.

\subsubsection{Crossover and Mutation}

The roulette method is adopted for selection. This selection strategy takes the proportion of fitness as the starting point so that the selection probability $p_{x}$ of individual $x$ is given as follows:

$$
\begin{gathered}
p_{x}=\frac{f_{x}}{\sum_{j=1}^{N} f_{j}} \\
f_{x}=\frac{k}{F_{x}}
\end{gathered}
$$

where $F_{x}$ is the fitness value of individual $x, N$ is the population, and $k$ is the coefficient. In this experiment, the crossover operator used single point crossover, the crossover probability is 0.7 , and the mutation probability is 0.01 . The evolutionary process of the GA is shown in Fig. 6, the error descent curve of the BP neural network 


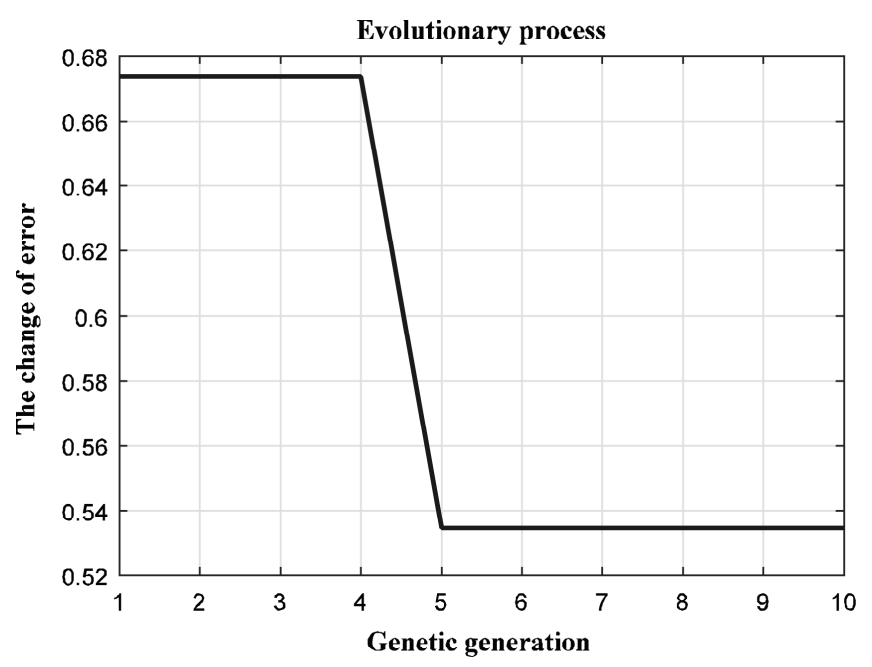

Figure 6. Evolutionary process.

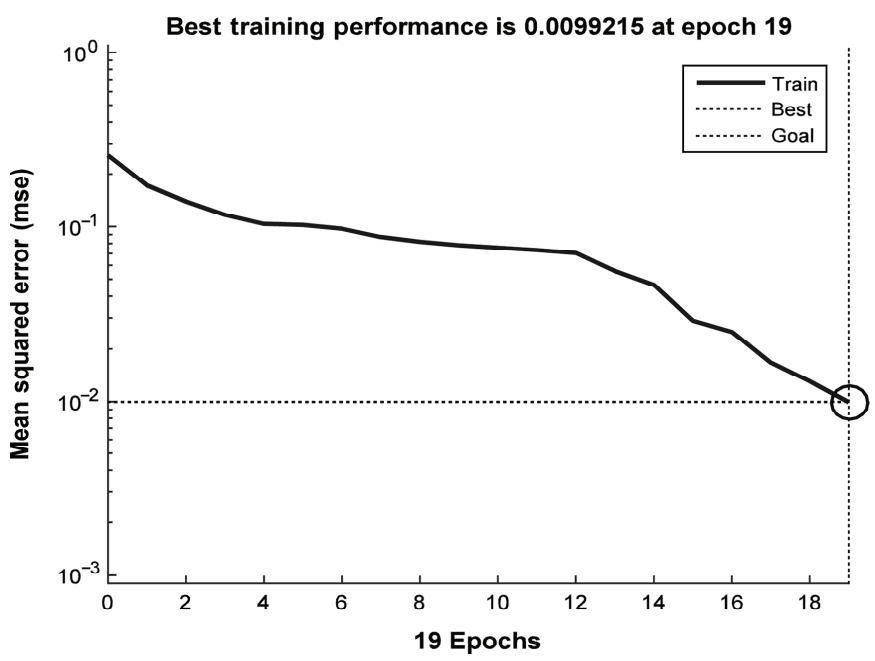

Figure 7. Error descent curve of GA-BP.

Table 3

Recognition Results

\begin{tabular}{|c|c|c|c|c|c|c|}
\hline \multirow{2}{*}{$\begin{array}{l}\text { Fault } \\
\text { Types }\end{array}$} & \multirow{2}{*}{$\begin{array}{c}\text { Number of } \\
\text { Samples }\end{array}$} & \multicolumn{4}{|c|}{$\begin{array}{l}\text { The Result of } \\
\text { Recognition }\end{array}$} & \multirow{2}{*}{$\begin{array}{l}\text { Accuracy } \\
\text { Rate (\%) }\end{array}$} \\
\hline & & N0 & N1 & F1 & F2 & \\
\hline N0 & 6 & 6 & 0 & 0 & 0 & 100 \\
\hline N1 & 7 & 0 & 7 & 0 & 0 & 100 \\
\hline F1 & 9 & 0 & 0 & 9 & 0 & 100 \\
\hline $\mathrm{F} 2$ & 22 & 0 & 0 & 0 & 22 & 100 \\
\hline
\end{tabular}

optimized by the GA is shown in Fig. 7, and the recognition results are shown in Table 3.

Comparing the results showing in Figs. 5 and 7, the GA-BP neural network is able to achieve the preset accuracy after 19 iterations, which is 17 fewer than the traditional BP neural network to yield a faster convergence rate. As it can be seen from Tables 2 and 3, the GA opti-

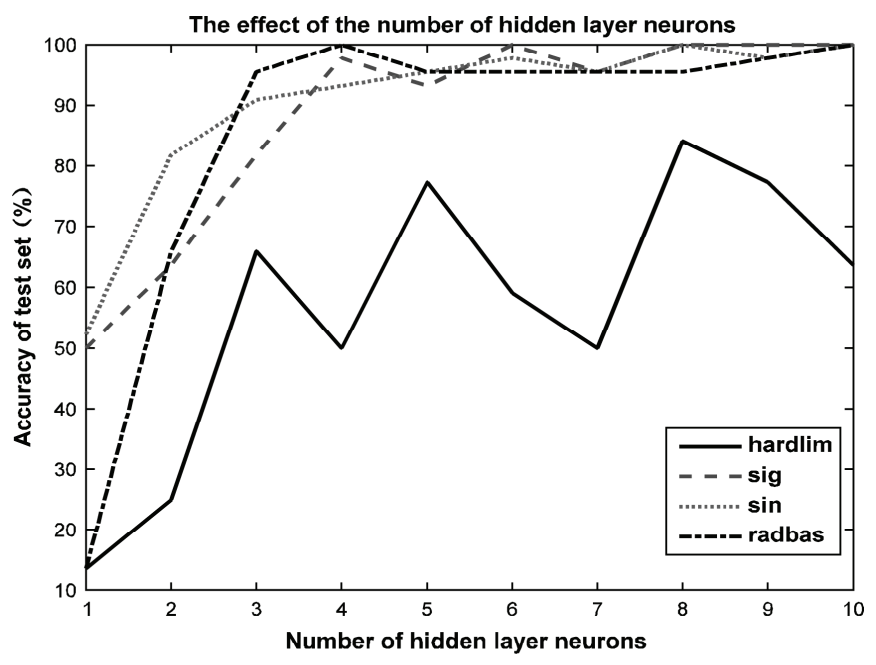

Figure 8. The effect of the number of hidden layer neurons.

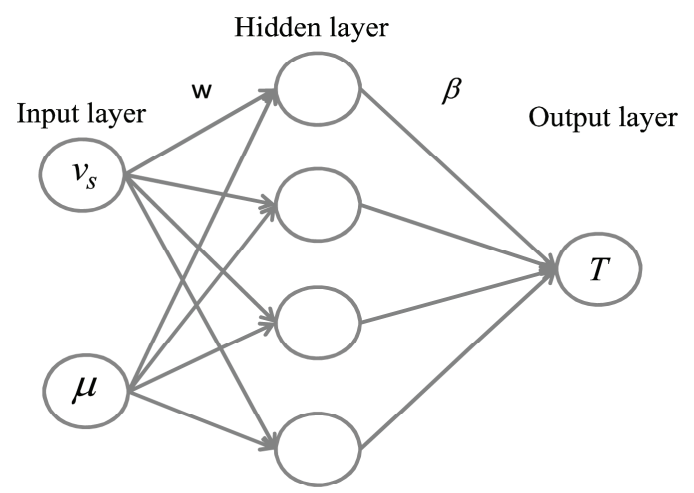

Figure 9. Schematic diagram of ELM structure.

Table 4

Recognition Results of ELM

\begin{tabular}{|c|c|c|c|c|c|c|}
\hline \multirow{2}{*}{$\begin{array}{l}\text { Fault } \\
\text { Types }\end{array}$} & \multirow{2}{*}{$\begin{array}{c}\text { Number of } \\
\text { Samples }\end{array}$} & \multicolumn{4}{|c|}{$\begin{array}{l}\text { The Result of } \\
\text { Recognition }\end{array}$} & \multirow{2}{*}{$\begin{array}{l}\text { Accuracy } \\
\text { Rate }(\%)\end{array}$} \\
\hline & & No & N1 & F1 & $\mathrm{F} 2$ & \\
\hline N0 & 6 & 6 & 0 & 0 & 0 & 100 \\
\hline N1 & 7 & 0 & 7 & 0 & 0 & 100 \\
\hline F1 & 9 & 0 & 0 & 9 & 0 & 100 \\
\hline $\mathrm{F} 2$ & 22 & 0 & 0 & 0 & 22 & 100 \\
\hline
\end{tabular}

mization method used in this study significantly improved the recognition accuracy of the $\mathrm{BP}$ neural network.

\subsection{ELM Simulation}

\subsubsection{Comparison of Different Activation Functions}

Equations (4)-(6) are used to study the effect of different activation functions and the number of hidden layer neurons on the accuracy rate. In the comparison testing, the four commonly used activation functions discussed above 
Table 5

Comparison Results

\begin{tabular}{|l|c|c|c|}
\hline Method & $\begin{array}{c}\text { Number of } \\
\text { Parameters }\end{array}$ & $\begin{array}{c}\text { Training } \\
\text { Time (s) }\end{array}$ & $\begin{array}{c}\text { Accurate Rate of } \\
\text { Test Set (\%) }\end{array}$ \\
\hline BPNN & 1 & 29.42411 & 84.09 \\
\hline GA-BP & 6 & 0.344946 & 100 \\
\hline ELM & 1 & 0.159189 & 100 \\
\hline
\end{tabular}

are used, and the number of hidden layer nodes is in the range from 1 to 10 , giving a total of $4 \times 10$ tests.

In the problem of adhesion state identification, the numbers of hidden layer neurons differ, and different activation functions exhibit varying performance (Fig. 8). Among the four functions, the hard-limit transfer activation function exhibits the lowest accuracy rate and most significant volatility; the three other types are slightly better, with the Sig and Sin functions indicating high recognition accuracy when the number of hidden layer neurons is high. The RBF function shows the smallest fluctuations and achieves an accuracy rate of $100 \%$ when the number of hidden layer neurons is set to 4 . The RBF is therefore selected as the activation function, and the ELM is given a $2 \times 4 \times 1$ structure. A schematic of the ELM structure is presented in Fig. 9. The experimental results are shown in Table 4.

From Table 4, it can be seen that our proposed ELM adhesion recognition method is able to accurately identify the four types of adhesion state, even when white Gaussian noise is added. This suggests that the ELM recognition model proposed in this study is applicable to the recognition of adhesion states.

\subsection{Comparison of the Three Recognition Methods}

We now discuss our experimental comparison of the training speed and accuracy rate of the ELM, the BP neural network, and the GA-BP neural network at different settings of the number of parameters. The results are shown in Table 5.

The accuracy of the ELM is equal to that of the GA$\mathrm{BP}$, but the GA-BP required more parameters to be set. The BP neural network had the worst performance. Its accuracy rate on the test set is also approximately $10 \%$ lower than those of the other two methods.

The comparison showed that the learning speeds of the ELM and GA-BP are significantly faster, whereas the accuracy rates remained sufficiently high. However, the training speed of the ELM is faster than that of the GA$\mathrm{BP}$, whereas fewer parameters needed to be set: only the number of hidden layer neurons had to be specified.

\section{Conclusion}

Accurate and timely automatic recognition of the adhesion state is the basis of effective adhesion control. Traditional recognition methods are slow and have low accuracy.
In this study, we proposed an approach to identify the adhesion state based on an ELM. This method allowed rapid and high accurate recognition rate of the adhesion state. The experimental results show that the ELM also achieved a good balance between time and accuracy in the presence of white Gaussian noise, which proves that this novel approach is applicable to the recognition of adhesion states.

\section{Acknowledgement}

This work was supported by the National Science Foundation of China (No. 61473117), Natural Science Foundation of Hunan Province (2016JJ5007) and Key Laboratory for Electric Drive Control and Intelligent Equipment of Hunan Province.

\section{References}

[1] W. Liao, H. Chen, W. Cai, et al., A novel active adhesion control design for high speed trains without vehicle speed measurement, 33rd China Control Conference (CCC), Nanjing, China, 2014, 221-226.

[2] K. Berntorp, Joint wheel-slip and vehicle-motion estimation based on inertial, GPS, and wheel-speed sensors, IEEE Transactions on Control Systems Technology, 24(3), 2016, 10201027.

[3] P. Pichlík, O. Zoubek, and J. Zděnek, Measuring device for measurement of train dynamic motion during wheel slip, 2014 IEEE Int. Conf. on Applied Electronics ( $A E)$, University of West Bohemia, Pilsen, Czech Republic, 2014, 247-250.

[4] S. Sadr, D.A. Khaburi, and J. Rodríguez, Predictive slip control for electrical trains, IEEE Transactions on Industrial Electronics, 63(6), 2016, 3446-3457.

[5] C.Y. Jae, Analysis of spectrum occupancy using machine learning algorithms, IEEE Transactions on Vehicular Technology, 83(331), 2015, 577-583.

[6] N. Li, X. Feng, and X. Wei, Optimized adhesion control of locomotive airbrake based on GSA-RNN, 2015 7th IEEE Int. Conf. on Intelligent Human-Machine Systems and Cybernetics (IHMSC), Hangzhou, China, 2015, 157-161.

[7] G. Zhuo and B. Wang, Structure designing of BP neural network in the application of reference velocity estimation, 2014 IEEE Int. Conf. on Mechatronics and Automation, Tianjin, China, 2014, 1481-1485.

[8] H. Chen, W. Cai, and Y. Song, Wheel skid prediction and antiskid control of high speed trains, 2014 IEEE Int. Conf. on Intelligent Transportation Systems (ITSC), Qingdao, China, 2014, 1209-1214.

[9] A. Suebsomran, Adaptive neural network control of electromagnetic suspension system, International Journal of Robotics and Automation, 29(2), 2014, 144-154.

[10] L. Tong, F. Zhang, Z.G. Hou, et al., BP-AR-based human joint angle estimation using multi-channel SEMG, International Journal of Robotics and Automation, 30(3), 2015, 227-237.

[11] G.B. Huang, H. Zhou, X. Ding, et al., Extreme learning machine for regression and multiclass classification, IEEE Transactions on Systems, Man, and Cybernetics, Part B (Cybernetics), 42(2), 2012, 513-529.

[12] T.T. Teo, T. Logenthiran, and W.L. Woo, Forecasting of photovoltaic power using extreme learning machine, 2015 IEEE Innovative Smart Grid Technologies-Asia (ISGT ASIA), Bangkok, Thailand, 2015, 1-6.

[13] Z. Bai, G.B. Huang, D. Wang, et al., Sparse extreme learning machine for classification, IEEE Transactions on Cybernetics, 44(10), 2014, 1858-1870.

[14] A. Mozaffari and N.L. Azad, Optimally pruned extreme learning machine with ensemble of regularization techniques and negative correlation penalty applied to automotive engine cold start hydrocarbon emission identification, Neurocomputing, 131, 2014, 143-156. 
[15] S. Salcedo-Sanz, C. Casanova-Mateo, A. Pastor-Sánchez, et al., Daily global solar radiation prediction based on a hybrid coral reefs optimization - extreme learning machine approach, Solar Energy, 105, 2014, 91-98.

[16] T. Koseki and T. Hara, Compensation of excessive angular momentum in a re-adhesion control of an electric train, 2015 Int. Conf. Electrical Systems for Aircraft, Railway, Ship Propulsion and Road Vehicles (ESARS), RWTH Aachen University, Aachen, Germany, 2015, 1-6.

[17] Y. Yao, S. Zhao, F. Xiao, and J. Liu, The effects of wheelset driving system suspension parameters on the re-adhesion performance of locomotives, Vehicle System Dynamics, 53(12), 2015, 1935-1951.

[18] G.B. Huang, Q.Y. Zhu, and C.K. Siew, Extreme learning machine: Theory and applications, Neurocomputing, 70(1), 2006, 489-501.

[19] H.-J. Rong, Y.-S. Ong, A.-H. Tan, and Z. Zhu, A fast prunedextreme learning machine for classification problem, Neurocomputing, 72(1), 2008, 359-366.

[20] G.B. Huang, Q.Y. Zhu, and C.K. Siew, Extreme learning machine: A new learning scheme of feed forward neural networks, Proc. 2004 IEEE Int. Joint Conf., Budapest, Hungary, 2004, 985-990.

[21] N. Jianjun, X. Yang, J. Chen, and S.X. Yang, Dynamic bioinspired neural network for multi-robot formation control in unknown environments, International Journal of Robotics and Automation, 30(3), 2015. DOI:10.2316/Journal.206.2015.3. 206-4217

[22] J. Zhang, F. Tian, S.X. Yang, Y. Liu, Z. Liang, and D. Wang, An intelligent and automatic control method for tobacco flue-curing based on machine learning, International Journal of Robotics and Automation, 31(6), 2016. DOI:10.2316/ Journal.206.2016.6.206-4697

[23] T. Huang, P. Yang, K. Yang, and Y. Zhu, Navigation of mobile robot in unknown environment based on $\mathrm{T}-\mathrm{S}$ neuro-fuzzy system, International Journal of Robotics and Automation, 30(4), 2015. DOI:10.2316/Journal.206.2015.4.206-4344

[24] F. Liu and L. Fei, Time-jerk optimal planning of industrial robot trajectories, International Journal of Robotics and Automation, 31(1), 2016. DOI:10.2316/Journal.206.2016.1.206-4055

[25] P. He and S. Dai, Real-time stealth corridor path planning for fleets of unmanned aerial vehicles in low-altitude penetration, International Journal of Robotics and Automation, 30(1), 2015, 60-69.

[26] F. Martın, L. Moreno, M.L. Munoz, et al., Initial population size estimation for a differential-evolution-based global localization filter, International Journal of Robotics and Automation, 29(3), 2014, 245-258.

[27] A. Maram, I. Chaari, A. Koubaa, et al., Global robot path planning using GA for large grid maps: Modelling, performance and experimentation, International Journal of Robotics and Automation, 31(6), 2016. DOI:10.2316/Journal.206.2016.6. 206-4602

\section{Biographies}

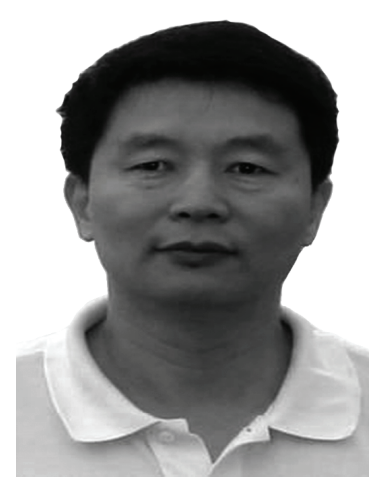

Changfan Zhang received his M.Sc. degree from Southwest Jiaotong University in 1989 and Ph.D. from Hunan University in 2001. Now, he is a professor in Hunan University of Technology. His main research direction is nonlinear control and applications.

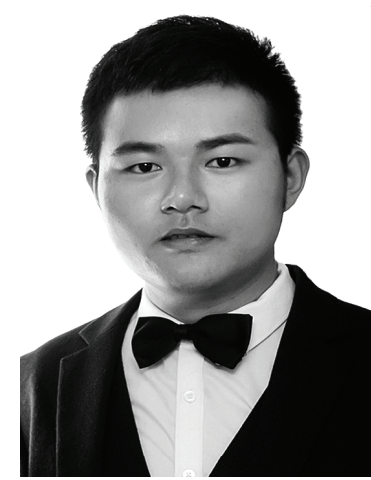

Xiang Cheng received his B.Sc. degree from Nanyang Institute of Technology in 2014. Now, he is an M.Sc. candidate in Hunan University of Technology. His main research direction is power transmission and fault diagnosis.

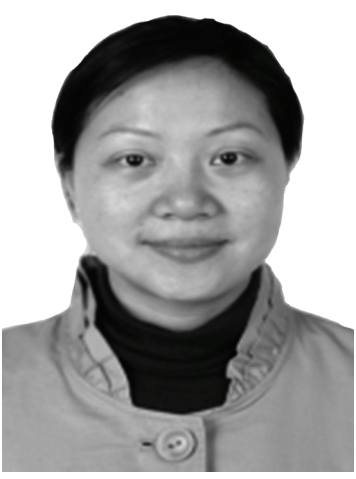

Jing $\mathrm{He}$ received his M.Sc. degree from Central South University of Forestry and Technology, in 2002, and Ph.D. from National University of Defense Technology, in 2009. She is currently a professor in Hunan University of Technology. Her main research direction is electro-mechanical system fault diagnosis.

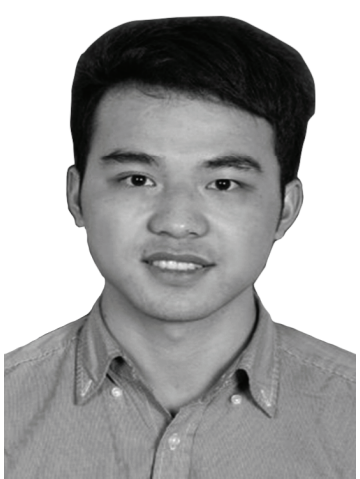

Guangwei Liu received his B.Sc. degree from Hunan Institute of Science and Technology in 2015. Now, he is an M.Sc. candidate in Hunan University of Technology. His main research direction is complex systems modelling. 